\title{
GUÍA PARA HACER UNA ENTREVISTA
}

Javier Araque Elaica

Magister

\section{Introducción}

Hacer una entrevista es una actividad absolutamente seria y responsable por parte del investigador/entrevistador como del entrevistado. La entrevista, es la comunicación interpersonal establecida entre el investigador y el sujeto de estudio a fin de obtener respuestas verbales a los interrogantes planteados sobre el problema de investigación propuesto; de manera que se convierte en una técnica de gran utilidad en la investigación cualitativa para obtener datos, comentarios y/o evidencias. Es claro que debe ser una conversación que se propone con un fin determinado distinto al simple hecho de conversar; de manera que se debe entender como un instrumento técnico que adopta la forma de un diálogo coloquial; su contenido es el soporte científico, técnico y/o legal de los resultados en la construcción de una investigación. En este orden de ideas, la entrevista es uno de los principales enfoques en la consecución de datos en la investigación cualitativa.

Conocemos una variedad de formas de hacer entrevistas con objetivos y principios diferentes. Al mismo tiempo, la entrevista se presenta con varias ventajas, prácticas y problemas diversos comunes a todas las opciones de su realización. Podemos estudiar la realización de entrevistas en niveles diferentes: teórico, epistemológico, ético y práctico. Una vez que la entrevista se ha realizado, le siguen varios pasos: las formas específicas de documentar lo que ha ocurrido en la entrevista individual llevan a necesidades y reglas para la transcripción. Los datos obtenidos demandan formas específicas de realización del análisis cualitativo. La realización de entrevistas trae consigo necesidades específicas de aumentar la calidad de la entrevista en general y su validez en particular, y finalmente de informar de lo que se dijo y cómo se analizó.

Este artículo trata detalladamente sobre la realización de entrevistas y se basa en la larga experiencia del autor para la realización de ellas. Supone una parte de la colección de investigación cualitativa que se dirige y tiene un sólido enfoque en la recogida de datos; pero también en cuestiones específicas sobre el análisis y la evaluación de esta forma específica de datos. Por consiguiente, algunos de los otros libros de la colección deberían ser útiles para complementar lo que se dice en este documento. En particular, se destacan los volúmenes de Gibbs (2007) sobre análisis de datos cualitativos y de Rapley (2007a) sobre análisis de discurso y de conversación que proporcionan más información sobre cómo analizar los datos cualitativos procedentes de entrevistas (y de otras fuentes).

Por su lado, el libro de Flick (2007a), entra más en los detalles de la planificación y el diseño de la investigación cualitativa (y de la realización de entrevistas) en general. Así, los problemas de calidad en la investigación cualitativa, que son también objeto de este documento, se debaten más 
detalladamente en el volumen sobre gestión de la calidad en la investigación cualitativa de Flick (2007a). En los otros libros de la colección de investigación cualitativa se resumen formas distintas o adicionales de recogida de datos. Por ejemplo, Angrosino (2007) presenta la observación participante y la etnografía (y también el uso de entrevistas en este contexto); Barbour (2007) detallada sobre los grupos de discusión como alternativa a las entrevistas individuales y Banks (2007), estudia el uso de datos visuales de manera más detallada.

Ahora bien, es de suma importancia que se entienda que si la entrevista es formal, es porque los encuentros ya fueron concertados y realizados según las reglas del entorno (normas, horarios, permisos, cultura) y en consecuencia se establece el concepto planeado. Las pautas a seguir en las entrevistas como los guiones de las mismas en todo caso y de manera previa es importante que el entrevistado conozca el cuestionario a fin de que el mismo no se sorprenda, tenga el tiempo de documentarse y/o no se sienta cohibido para dar sus respuestas.

Por lo anterior, para llegar a un feliz término en el propósito investigativo se recomienda tener presente ocho (8) pasos:

\section{Paso 1. Presentación}

La entrevista ocupa un lugar privilegiado en el centro de toda negociación humana, pues supera en mucho los conceptos de una charla o conversación; de un mero interrogatorio o incluso, de una disertación, dado que la entrevista se plantea como una forma oral de comunicación interpersonal, que tienen como finalidad obtener información en relación con un objetivo, permitiendo que sus participantes tengan la oportunidad de vivenciar la dinámica de la reciprocidad.

En correspondencia con lo anterior, pida el favor al entrevistado, que le permita establecer un tiempo y lugar adecuado para realizar la entrevista congruente con el objeto de la investigación; además, explique el propósito académico de la entrevista. Antes de iniciar, "y que quede grabado", por favor pida al entrevistado que se presente e informe sus nombres y apellidos, su cargo y otra información que permita establecer la importancia de sus respuestas en el marco de la investigación. En todo caso, que el entrevistado tenga claro que ese proceso es una técnica de obtención de información mediante el diálogo mantenido en el encuentro formal o planeado según se halla acordado, entre una o más personas entrevistadoras y una o más entrevistadas, en el que se transforma y sistematiza la información conocida por las mismas

\section{Tema central}

Al momento de realizar la entrevista el (los) entrevistador (es) debe (n) tener presente y permanentemente el título de la investigación y/o trabajo de grado; para que oportunamente se recuerden y tengan presente los objetivos específicos a alcanzar; ya que justamente la entrevista es parte de uno de ellos; por consiguiente, es importante delimitar el tema eje, en el que girará la entrevista, ya que focalizar la atención en un punto determinado se puede obtener información precisa y de mayor calidad que permita satisfacer la necesidad de la técnica de investigación 


\section{Preguntas orientadoras}

En ocasiones es mejor formular preguntas de acuerdo con la información que se va generando, así como el ritmo de la entrevista; sin embargo, en cualquiera de los casos siempre es recomendable establecer una serie de preguntas guía para evitar que se pierdan objetivo central de la entrevista, El proceso de entrevistar a alguien se perfecciona con el tiempo, mediante la permanente reevaluación de métodos y técnicas. Con cada entrevista, el investigador fortalece sus habilidades y mejora su capacidad para plantear las preguntas y registrar las respuestas en forma precisa, lógica y completa.

Las deficiencias en las entrevistas pueden generar una impresión desfavorable y afectar la calidad de la investigación

Cuando el investigador se hace una pregunta desde cualquier tema, es porque desea tener una respuesta a un problema o una necesidad, que lo lleve por un camino racional y ordenado de las cosas o situaciones dadas, acerca del problema planteado. Sin unas buenas preguntas orientadoras resulta difícil diseñar y llevar a cabo un estudio; sino se tiene claro sobre lo que se está investigando. Por ello la realidad que nos rodea porque es de esa realidad, de la práctica cotidiana, de las vivencias, lecturas, de la asistencia a eventos de donde surgen la mayoría de preguntas (de investigación); resulta difícil que un tema de investigación surja de repente en la mente del observador o investigador. Lo más probable es que la experiencia acumulada en el ejercicio profesional, en determinado campo, lo pueda orientar hacia una buena observación o hacia un proyecto de investigación

El dicho, que "la experiencia es la madre de la ciencia" tiene sentido en la postura crítica que mantiene el investigador ante una realidad que lo circunda; esta actitud indagante, es una primera premisa de una investigación, donde debe también existir un interés claro de llevar a cabo esta actividad.

Bajo una postura de observador crítico, de una realidad dada, se actúa buscando respuestas a necesidades evidenciadas en los diferentes contextos socioeconómicos; de manera que para la formulación de una pregunta se deben tener en cuenta los elementos tiempo, espacio y tema; sin dejar de lado aspectos como actualidad, viabilidad; la misma del proyecto de investigación debe también considerar a la hora de formular la pregunta, ¿si se dispone de los recursos humanos y los materiales adecuados para la realización de la misma?, el tiempo que requiere la investigación, ¿es proporcionado a nuestra capacidad?, ¿se cuenta con la formación metodológica para el proyecto que se pretende abordar?

Pertinencia: el estudio o trabajo que se realizará, debe ser relevante y estar contextualizado para el área o disciplina y suscita preguntas como: ¿los conocimientos que se obtendrán beneficiarán al área de la educación física (profesores y estudiantes), a la comunidad o a la sociedad? ¿los resultados resultarán de aplicación práctica o importancia teórica?

Operatividad: toda pregunta debe estar formulada en términos operativos, es decir, que la misma debe sugerir el camino que ha de seguir la investigación y orientar la metodología a seguir en el diseño y ejecución del estudio. 


\section{Etapas de formulación de la pregunta}

3.1.Definir el tema: es el inicio de la labor de un buen investigador y tiene que ver con las preocupaciones, intereses, motivaciones e inquietudes del (os) investigador (es). Preguntas como ¿qué situación concreta, problema, evento, lugar y/o institución interesa conocer?

3.2.Delimitar el tema: esto significa enfocar en términos concretos nuestra área de interés, especificar sus alcances y determinar límites. Se trata de decidir hasta dónde llega la investigación a realizar, qué variables se van a tener en cuenta en la investigación. Por variable se entiende las características o cualidades de la realidad susceptible de calificar con diferentes valores; es decir, que puede variar. La edad, por ejemplo, es una variable porque puede ser de cinco, veinte cuarenta años; varía, asume diferentes valores. Otras variables pueden ser el sexo, nivel escolar, institución educativa y estrategias de enseñanza. Elegido y delimitado el tema de investigación, se debe seguir precisando el problema, pensando y escribiendo preguntas como: ¿qué referentes teóricos se necesitan para comprender mejor el tema de investigación?, ¿qué se conoce acerca del ámbito a investigar?, ¿se rescatan conocimientos previos?, ¿cuáles son los escenarios y las personas que forman parte de la investigación? y ¿qué metodología resulta más útil para indagar sobre los interrogantes?

3.3.Determinar los objetivos de la investigación: las preguntas orientadoras de la entrevista deben ir de la mano con el objetivo de la misma. Las preguntas ayudan a definir los objetivos de la investigación. Estos últimos son guías y durante todo el desarrollo se deben tener presentes para no desviar la entrevista.

\section{Postura y lenguaje corporal}

Para la entrevista es importante que entiendan que un factor a tomar en cuenta, es el lenguaje corporal; tanto el del entrevistador como el del entrevistado. Esto determina el ritmo de la entrevista, que en mayor o menor medida, influirá en la calidad de la información obtenida. Aunque no se hace conscientemente, cuando se está hablando, se sigue comunicando con el lenguaje corporal o no verbal; con los movimientos y gestos se exterioriza lo que se piensa internamente sin ser conscientes de que lo se hace.

El entrevistador puede, gracias al contacto personal, obtener información muy útil y veraz sobre el objeto de la entrevista según su lenguaje verbal y corporal; por tanto, tan importante es lo que el entrevistador dice de sí mismo como la manera en que lo expresa a través de los signos del lenguaje no verbal, ya que el lenguaje corporal no miente. Con las expresiones reflejamos lo que se piensa, pues es muy difícil controlar a la vez lo que se dice y expresa.

Muchas veces cuando se afirma algo verbalmente, esa comunicación no corresponde con lo que se transmite con los movimientos o gestos. Queda claro que no se está diciendo la verdad porque se vislumbran contradicciones entre lo que se dice y lo que se hace.

Por ello, es muy importante conocer cómo utilizar las posturas del cuerpo, los gestos del rostro o los movimientos de las manos para transmitir una imagen positiva del entrevistador ante la persona que aceptó ser entrevistado.

Es importante reforzar la primera impresión ya que va a marcar la dirección y tono de la entrevista, es vital y para ello lo esencial desde el punto de vista del lenguaje corporal es saber dar la mano apropiadamente. Cuando se da la mano se mira a los ojos al entrevistador y dibuja una sonrisa de empatía, el apretón tiene que ser firme, pero no muy alargado en el tiempo.

Usar los ojos para comunicarse. Se debe intentar mirar al entrevistado directamente a los ojos, pero sin incomodar. Recordar que una mirada a los ojos puede ser interpretada como una señal de agresión y una mirada de costado es interpretada como una mentira. La mirada 
debe ser empática, que proyecte entusiasmo, interés y confianza.

Sonreír. La sonrisa natural, sin excederse, es esencial en el lenguaje corporal para mostrar simpatía, se trata de sonreír no de reírse todo el tiempo y de todo; se recomienda entrenar en un espejo la mejor sonrisa para no parecer fingida.

Controlar el cabello. A nadie le gusta alguien que permanentemente esté acomodándose el cabello o que caiga constantemente sobre la cara. Cuando una persona está todo el tiempo acomodándose el cabello, se puede interpretar como una falta de respeto, porque da a entender que no le interesa lo que te dice el interlocutor; que el entrevistador está pensando en otras cosas. Un buen corte es esencial; por lo que no se recomienda entrevistar con el cabello recogido y si lo lleva largo y por supuesto bien peinado, cómodo para que no sea una distracción en la entrevista.

Proyecta confianza con la postura del cuerpo. Esto es vital y tal vez recordará los consejos de sentarse derecho con la espalda recta. No apoyarse sobre la mesa y por supuesto, nada de recostarse hacia atrás en la silla; además, la cabeza debe estar erguida y derecha y tratar de sentarse un poco al borde de la silla para demostrar interés al entrevistado, pero sobre todo no sentarse en la silla excesivamente rígido, como paralizado, al contrario, correcto y relajado.

Expresiones faciales positivas. El rostro sirve para comunicar expresiones faciales de asombro o de duda; se debe tratar de ser positivo con las expresiones faciales, más allá del simple sonreír.

Evitar y/o controlar los tics nerviosos. Es imposible no tener algo de nervios al hacer una entrevista y a veces se tienen algunos descuidos como jugar con un bolígrafo, una hoja o una carpeta, jugar con los anillos, el reloj y jugar con los dedos. Se debe identificar esos tics y tratar de controlarlos. Si se juega con anillos o joyas no portarlos en ese momento, dejar el bolígrafo encima de la mesa; aunque sin duda la mejor forma de controlar los tics es intentar relajarse antes de la entrevista, sentirse cómodo. Se debe de proyectar una imagen de interés hacia el entrevistado y estar distraído con tics demuestra lo contrario. Transmite dinamismo y simpatía con el cuerpo. No se debe mostrar conflictivo, recordar que el entrevistado busca a alguien que va a mostrar capacidad de trabajo en equipo y convivencia diaria.

Usar las manos de forma apropiada para enfatizar los puntos fuertes y las cosas más importantes a destacar.

\section{Registro de la entrevista}

Para hacer una entrevista se debe contar con ayudas audiovisuales cuyas características de batería, memoria y versatilidad permitan y motiven confianza y garantía de conservar la información. Si es un celular, revisar que haya suficiente espacio y batería al igual que con una videocámara, revisar siempre el audio, realizar grabación de la entrevista en forma audiovisual; grabar la entrevista es una regla de oro, siempre en común acuerdo con el entrevistado, a fin de evitar controversias legales; pero principalmente, para poder retomar información específica sin necesidad de confiar de más en la memoria. Igualmente, el uso de una agenda de notas y un bolígrafo junto con fotografías complementarias, garantizará siempre calidad en la información.

\section{Contextualización e investigación previa}

En correspondencia con lo desarrollado, investigado y vivido en un taller les dan suficientes elementos para realizar una investigación previa relacionada con el tema central y con la trayectoria o influencia de la persona a entrevistar; obteniendo así las suficientes armas para generar una entrevista exitosa y fuera de serie.

\section{Despedida y cierre}

Finalizada la entrevista, el entrevistador debe asegurarse de que audiovisualmente quede grabado: nombres y apellidos del entrevistador, fecha de la entrevista, lugar de la entrevista, descripción del evento de la entrevista y descripción de la investigación para la que se hace la entrevista. 


\title{
8. Sistematización
}

Mediante un programa de traducción o un digitador, el total de la entrevista debe sistematizarse en medio físico, sin omitir nada ni resumir y/o dar opiniones o conceptos del transcriptor.

\author{
Anexo \\ MODELO/GUÍA PARA LEVANTAR UNA FICHA AUDIOGRÁFICA \\ Fuente: diseñada por Mg Javier Araque Elaica
}

\begin{tabular}{|l|l|}
\hline AUDIO VIDEO & Título de la entrevista relacionado con la investigación \\
\hline \hline ENTREVISTADO & $\begin{array}{l}\text { Datos del entrevistado, teléfono, email, cargo, dirección, en- } \\
\text { tre otros }\end{array}$ \\
\hline ENTREVISTADOR & Datos del entrevistado, teléfono, email, dirección, entre otros \\
\hline CIUDAD Y FECHA & $\begin{array}{l}\text { Tema de la entrevista, relacionado con el objetivo específico } \\
\text { correspondiente, }\end{array}$ \\
\hline TEMA & \\
\hline FECHA ENTREVISTA & (Ver prólogo, introducción, índice entre otros) \\
\hline BREVE DESCRIPCIÓN
\end{tabular}

\begin{tabular}{|l|l|l|}
\hline TIEMPO & COMDIO-VIDEO \\
\hline 00,01 & $\begin{array}{l}\text { Finalizada la entrevista el en- } \\
\text { trevistador debe asegurarse } \\
\text { de que audiovisualmente } \\
\text { quede grabado: Nombres y } \\
\text { Apellidos del entrevistador, } \\
\text { fecha de la entrevista, lugar } \\
\text { de la entrevista, descripción } \\
\text { del evento de la entrevista y } \\
\text { descripción de la investiga- } \\
\text { ción para la que se hace la } \\
\text { entrevista. } \\
\text { Siendo las 06:01 se da por }\end{array}$ & Prestación del entrevis- \\
terminada la entrevista & tado \\
\hline
\end{tabular}

\section{Comentarios generales y perspectiva del entrevistador}

(Complemente con el número de hojas que considere necesario)

\section{Glosario}

Entrevista: Es un procedimiento del investigador donde se conjugan elementos técnicos, destrezas cognitivas y emocionales, además de habilidades de comunicación, cuyo objetivo es obtener la mayor cantidad de información útil posible mediante la adecuada formulación de preguntas.

Entrevistador: Quien pregunta, aplica o desarrolla la entrevista. Entrevistado: Quien contesta a permite que se le pregunte.

\section{Bibliografía}

Breakwell, G. (2002). Cómo realizar entrevistas con éxito. Barcelona: Editorial Gestión 2000.

Acevedo, A., y López A. (1986). El proceso de la entrevista: conceptos y modelos. Editorial Limusa.

Kvale, S. (2011). Las entrevistas en investigación cualitativa. Editorial Morata. 